\title{
LOCAL MONOTONICITY OF MEASURES SUPPORTED BY GRAPHS OF CONVEX FUNCTIONS
}

\author{
ROBERT ČERNÝ
}

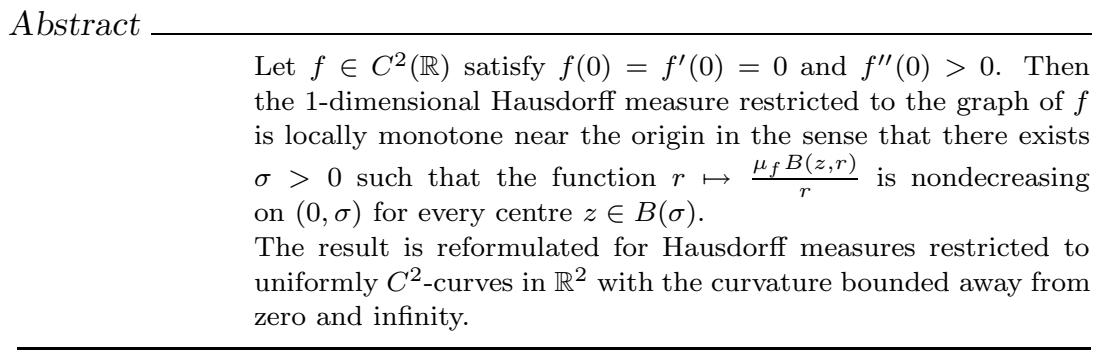

\section{Introduction}

In this paper, we are interested in local monotonicity of measures in $\mathbb{R}^{2}$. Study of monotone measures is motivated by open problems on existence and regularity of minimal surfaces. For the way, how the Monotonicity Formula is used in current proofs, see for example [5].

Definition 1.1. Let $\mu$ be a Radon measure on $\mathbb{R}^{n}$ and $k \in \mathbb{N}$. We say that $\mu$ is $k$-monotone if the function $r \mapsto \frac{\mu B(z, r)}{r^{k}}$ is nondecreasing on $(0, \infty)$ for every $z \in \mathbb{R}^{n}$. Instead of 1 -monotone, we simply write monotone.

It is natural to ask whether there exists a monotone measure with non unique tangential behaviour. Such a measure was first given by Kolár. He observed that non monotonicity of a measure can be sometimes compensated by adding a measure that is abundant in monotonicity. Therefore, he carefully constructed a measure with bad tangential behaviour supported by a curve in $\mathbb{R}^{2}$, which was not too much non monotone, and then adding a measure absolutely continuous with respect to the Lebesgue measure he obtained the demanded measure.

2000 Mathematics Subject Classification. 53A10, 49Q15, 28A75.

Key words. Monotone measure, monotonicity formula. 
Nevertheless, it would be useful to have a monotone measure with bad tangential behaviour satisfying some additional density assumptions (the density should be bounded from bellow by one on the support and it should be also bounded from above by $1+\varepsilon$, for any given $\varepsilon>0$, which would show that Allard's regularity theorem, see [1], cannot be generalized).

As a candidate for such a measure, there was considered the 1-dimensional Hausdorff measure restricted to a symmetrical pair of logarithmic spirals $(\Gamma(t)=(t \cos (a \ln |t|), t \sin (a \ln |t|)), t \in \mathbb{R} \backslash\{0\}, \Gamma(0)=(0,0))$. Such measures have the density $\theta_{z}^{1} \mu_{a}=1$ for $z \in \operatorname{spt} \mu_{a} \backslash\{0\}$ and $\theta_{0}^{1} \mu_{a}=\frac{\mu B(0, r)}{r}=\sqrt{1+a^{2}}$, for every $r \in(0, \infty)$.

However, Huovinen showed that for every $a \neq 0, \mu_{a}$ is not monotone. On the other hand, using the Taylor series and the self similarity of the support Kirchheim proved that $\mu_{a}$ is locally monotone. More precisely, there is a constant $c_{a}>0$ depending only on $a \neq 0$ such that the function $r \mapsto \frac{\mu B(z, r)}{r}$ is nondecreasing on $\left(0, c_{a}|z|\right), z \in \mathbb{R}^{2} \backslash\{0\}$. Hence, the Kolár compensation method gives that for every $a \neq 0$, there is a finite number of lines $L_{1}, \ldots, L_{n_{a}}$ going through the origin such that the measure $\mu_{a}+\mathcal{H}^{1}\left\llcorner\bigcup_{i=1}^{n_{a}} L_{i}\right.$ is monotone.

Kirchheim's results were improved in [2], where the lower estimates for $c_{a}$ were found. This enabled to prove that for sufficiently small $|a| \neq$ 0 , only two lines are enough as a compensation for $\mu_{a}$. Because of the self similarity of logarithmic spirals it is easy to see that a single line is not sufficient.

In paper [3], there is even given an example of a family of measures $\mu_{a, c}$ in $\mathbb{R}^{3}$ with bad tangential behaviour that can be compensated by a single line. Thus, the density of the obtained monotone measure $\mu_{a, c}+\mathcal{H}^{1}\llcorner L$ is between 1 and $2+\varepsilon$ on the support.

We see that the local monotonicity is not only a poor weak version of the monotonicity, but it also plays an important role when used together with the Kolár compensation method.

In this paper, we are interested in two notions of local monotonicity in $\mathbb{R}^{2}$. Although, for applications, is it useful to have uniform local monotonicity, i.e. $r \mapsto \frac{\mu B(z, r)}{r}$ nondecreasing on $(0, \sigma)$ for some $\sigma>0$ independent of a centre $z \in \mathbb{R}^{2}$, or at least on large sets of centres, the example of the logarithmic spirals also shows that it is sometimes useful to study pointwise local monotonicity too.

The following section is devoted to the main result concerning the uniform local monotonicity of measures supported by graphs of convex $C^{2}$-functions with the second derivative bounded away from zero. 
In the third section, we reformulate the main result for uniformly $C^{2}$-curves in $\mathbb{R}^{2}$ with the curvature bounded away from zero and infinity.

Finally, in the fourth section, we give an example showing that even if the function $f$ is strictly convex and $C^{\infty}$, then we generally lose even the pointwise local monotonicity.

We refer to [4] and [5] for other information about the geometry of measures and the Monotonicity Formula.

Notation. The scalar product of $x, y \in \mathbb{R}^{n}$ is denoted by $x \cdot y$ and the Euclidean norm of $x$ is denoted by $|x|$. The 1-dimensional Hausdorff measure is denoted by $\mathcal{H}^{1}$. And $\mathcal{H}^{1}\llcorner A$ denotes its restriction to a Borel set $A$. Further

$B(z, r)=\left\{x \in \mathbb{R}^{n}:|x-z| \leq r\right\} \quad$ and $\quad S(z, r)=\left\{x \in \mathbb{R}^{n}:|x-z|=r\right\} ;$

when $z=0$, we simply write $B(r)$ and $S(r)$. For $h \in \mathbb{R}, x>0$ and an even function $f: \mathbb{R} \rightarrow \mathbb{R}$, we define $z_{h}=(0, h)$ and $r_{h}(x)=$ $\left|(x, f(x))-z_{h}\right|=\sqrt{x^{2}+(f(x)-h)^{2}}, \eta(x)=\arctan \left(f^{\prime}(x)\right)$ and $\varphi_{h}(x)=$ $\arctan \left(\frac{f(x)-h}{x}\right)$.

We say that a Radon measure $\mu$ is monotone at $(z, r)$ if

$$
\underline{\mathrm{D}}_{\mathrm{r}} \frac{\mu B(z, r)}{r} \geq 0
$$

where $\underline{\mathrm{D}}_{\mathrm{r}} f(r)=\liminf _{\delta \rightarrow 0} \frac{f(r+\delta)-f(r)}{\delta}$.

Finally, for a Borel function $f:(a, b) \rightarrow \mathbb{R},-\infty \leq a<b \leq \infty$, let

$$
\mu_{f}=\mathcal{H}^{1}\llcorner\{(x, f(x)): x \in \mathbb{R}\}
$$

and for an absolutely continuous curve $\gamma:[a, b] \rightarrow \mathbb{R}^{n},-\infty<a<b<\infty$, we define

$$
\mu_{\gamma}(A)=\int_{\{t \in(a, b): \gamma(t) \in A\}}|\mathrm{D} \gamma(t)| d t
$$

\section{Sufficient conditions for local monotonicity}

In this section, our main result is

Theorem 2.1. Let $f \in C^{2}(\mathbb{R}), f(0)=f^{\prime}(0)=0$ and $f^{\prime \prime}(0)>0$. Then there exists $\sigma>0$ such that $r \mapsto \frac{\mu_{f} B(z, r)}{r}$ is nondecreasing on $(0, \sigma)$ for every centre $z \in B(\sigma)$.

The proof of Theorem 2.1 is based on 
Proposition 2.2. Let $\delta \in\left(0, \frac{1}{20}\right]$ and $f \in C^{2}((-\delta, \delta), \mathbb{R})$ be a function satisfying $f(0)=f^{\prime}(0)=0, f^{\prime \prime}(x) \in\left[\frac{19}{20}, \frac{21}{20}\right]$ on $(-\delta, \delta)$. Then $r \mapsto$ $\frac{\mu_{f} B\left(z_{h}, r\right)}{r}$ is nondecreasing on $(0, \delta)$ for every $h \in \mathbb{R}$.

The following lemma enables us to use Proposition 2.2 even when the centre $z$ is not restricted to the $y$-axis.

Lemma 2.3. Let $f \in C^{2}(\mathbb{R}), f(0)=f^{\prime}(0)=0, f^{\prime \prime}(0)=1$. Then there exists $\delta>0$ such that for every $x_{0} \in(-\delta, \delta)$, the function

$\tilde{f}(x)=\frac{1}{\sqrt{1+f^{\prime 2}\left(x_{0}\right)}}\left(f\left(x_{0}+\frac{x}{\sqrt{1+f^{\prime 2}\left(x_{0}\right)}}\right)-f\left(x_{0}\right)-\frac{f^{\prime}\left(x_{0}\right) x}{\sqrt{1+f^{\prime 2}\left(x_{0}\right)}}\right)$ is defined on $(-\delta, \delta)$ and satisfies $\tilde{f}(0)=\tilde{f}^{\prime}(0)=0, \tilde{f}^{\prime \prime}((-\delta, \delta)) \subset$ $\left[\frac{19}{20}, \frac{21}{20}\right]$.

Proof: Since $f^{\prime \prime}$ is continuous, there is $\tilde{\delta}>0$ such that $f^{\prime \prime}(t) \in\left[\frac{39}{40}, \frac{21}{20}\right]$ on $(-\tilde{\delta}, \tilde{\delta})$. Let us set $\delta=\min \left(\frac{1}{2} \tilde{\delta}, \frac{1}{20}\right)$. Then the assertion follows from the definition of $\tilde{f}$ and

$$
\tilde{f}^{\prime \prime}(x)=f^{\prime \prime}\left(x_{0}+\frac{x}{\sqrt{1+f^{\prime 2}\left(x_{0}\right)}}\right)\left(1+{f^{\prime 2}}^{2}\left(x_{0}\right)\right)^{-\frac{3}{2}} .
$$

We prove Proposition 2.2 showing that $\mu_{f}$ is monotone at $(z, r)$ for every centre $z_{h}, h \in \mathbb{R}$ and for every radius $r \in(0, \delta)$.

In the following, let $f$ and $\delta \in\left(0, \frac{1}{20}\right]$ satisfy the assumptions of Proposition 2.2. Since for $h \in \mathbb{R}$ and $r>0$, we have

$$
\mu_{f(\cdot)} B\left(z_{h}, r\right)=\frac{1}{2}\left(\mu_{f(|\cdot|)} B\left(z_{h}, r\right)+\mu_{f(-|\cdot|)} B\left(z_{h}, r\right)\right)
$$

and

$$
\mu_{f} B\left(z_{h}, r\right)=\left.\mu_{f}\right|_{\left\{t \in \mathbb{R}:(t, f(t)) \in B\left(z_{h}, r\right)\right\}} B\left(z_{h}, r\right),
$$

we suppose, without loss of generality, that $f$ is an even function satisfying $0 \leq f^{\prime \prime}(x) \leq \frac{21}{20}$ on $\mathbb{R}$.

The idea of the proof of Proposition 2.2 uses the Taylor expansion. The following Lemma 2.4 gives us a suitable parameterization. 
Lemma 2.4. Assume $x \in[0, \delta)$ and $h \leq \frac{20}{21}$. Then

$$
S\left(z_{h}, r_{h}(x)\right) \cap \operatorname{spt} \mu_{f}=\{(x, f(x)),(-x, f(x))\} .
$$

Moreover, if $x \in(0, \delta)$, then $\left.\frac{\partial r_{h}(t)}{\partial t}\right|_{t=x} \in(0, \infty)$ and

$$
\left.\frac{\partial \mu_{f} B\left(z_{h}, r\right)}{\partial r}\right|_{r=r_{h}(x)}=\frac{2}{\cos \left(\eta(x)-\varphi_{h}(x)\right)} .
$$

Proof: $S\left(z_{h}, r_{h}(x)\right) \cap \operatorname{spt} \mu_{f} \supset\{(x, f(x)),(-x, f(x))\}$ holds trivially. On the other hand, since $h \leq \frac{20}{21} \leq \sup _{t \in(0, \infty)} \frac{1}{f^{\prime \prime}(t)} \leq \sup _{t \in(0, \infty)} \frac{t}{f^{\prime}(t)}$, we have

$$
\frac{\partial r_{h}(t)}{\partial t}=\frac{t+(f(t)-h) f^{\prime}(t)}{r_{h}(t)}>\frac{t-h f^{\prime}(t)}{r_{h}(t)} \geq 0
$$

on $(0, \infty)$. Hence, the continuous function $t \mapsto r_{h}(t)$ is increasing on $[0, \infty)$.

Finally, let $F(x, r)=x^{2}+(f(x)-h)^{2}-r^{2}$. The Implicit Function Theorem gives

$$
\begin{aligned}
\left.\frac{\partial \mu_{f} B\left(z_{h}, r\right)}{\partial r}\right|_{r=r_{h}(x)} & =\left.\frac{\partial \mu_{f} B\left(z_{h}, r_{h}(x)\right)}{\partial x} \frac{\partial x}{\partial r}\right|_{r=r_{h}(x)} \\
& =\left.2 \sqrt{1+f^{\prime 2}(x)}(-1) \frac{\frac{\partial F}{\partial r}}{\frac{\partial F}{\partial x}}\right|_{r=r_{h}(x)} \\
& =2 \frac{\sqrt{1+f^{\prime 2}(x)} r_{h}(x)}{x+(f(x)-h) f^{\prime}(x)} \\
& =2 \frac{\left|\left(1, f^{\prime}(x)\right)\right||(x, f(x)-h)|}{\left(1, f^{\prime}(x)\right) \cdot(x, f(x)-h)} \\
& =\frac{2}{\cos \left(\eta(x)-\varphi_{h}(x)\right)}
\end{aligned}
$$

Proof of Proposition 2.2: Suppose $h \in \mathbb{R}$ and $r \in(0, \delta)$. If $h \geq \frac{20}{21}$, then for every $x \in(0, \delta)$, we have

$$
r_{h}(x) \geq h-f(x) \geq h-f(\delta) \geq \frac{20}{21}-\frac{21}{20} \delta^{2}>\delta>r .
$$

Hence $B\left(z_{h}, r\right) \cap \operatorname{spt} \mu_{f}=\emptyset$ and the proof is trivial.

If $h \leq \frac{20}{21}$ and $S\left(z_{h}, r\right) \cap \operatorname{spt} \mu_{f} \subset\{(0,0)\}$, then again $\mu_{f} B\left(z_{h}, r\right)=0$ by Lemma 2.4 . 
In the remaining case, there is unique $x \in(0, \delta)$ such that $r=r_{h}(x)$. Further, Lemma 2.4 gives

$$
\begin{aligned}
\frac{\partial}{\partial r} & \left.\frac{\mu_{f} B\left(z_{h}, r\right)}{r}\right|_{r=r_{h}(x)} \\
& =\left.\frac{1}{r^{2}}\left(\frac{\partial \mu_{f} B\left(z_{h}, r\right)}{\partial r} r-\mu_{f} B\left(z_{h}, r\right)\right)\right|_{r=r_{h}(x)} \\
& =\left.\frac{1}{r^{2}}\left(\frac{2}{\cos \left(\eta(x)-\varphi_{h}(x)\right)} \frac{x}{\cos \left(\varphi_{h}(x)\right)}-\mu_{f} B\left(z_{h}, r\right)\right)\right|_{r=r_{h}(x)} \\
& =\left.\frac{1}{r^{2}}\left(\frac{4 x}{\cos \left(\eta(x)-2 \varphi_{h}(x)\right)+\cos (\eta(x))}-\mu_{f} B\left(z_{h}, r\right)\right)\right|_{r=r_{h}(x)} \\
& \geq\left.\frac{1}{r^{2}}\left(\frac{4 x}{1+\cos (\eta(x))}-\mu_{f} B\left(z_{h}, r\right)\right)\right|_{r=r_{h}(x)} \\
& =\frac{1}{r_{h}(x)^{2}}\left(\frac{4 x \sqrt{1+f^{\prime 2}(x)}}{\sqrt{1+f^{\prime 2}(x)}+1}-2 \int_{0}^{x} \sqrt{1+f^{\prime 2}(t)} d t\right) .
\end{aligned}
$$

Finally, using the Taylor expansion we obtain

$$
\begin{aligned}
4 x \sqrt{1+f^{\prime 2}(x)} & \geq 2 x \sqrt{1+f^{\prime 2}(x)}+2 x+\frac{1}{3}\left(\frac{21}{20}\right)^{2} x^{3}\left(\sqrt{1+f^{\prime 2}(x)}+1\right) \\
& =2\left(x+\frac{1}{6}\left(\frac{21}{20}\right)^{2} x^{3}\right)\left(\sqrt{1+f^{\prime 2}(x)}+1\right) \\
& \geq 2 \int_{0}^{x} \sqrt{1+f^{\prime 2}(t)} d t\left(\sqrt{1+f^{\prime 2}(x)}+1\right) .
\end{aligned}
$$

Therefore, $\mu_{f}$ is monotone at $\left(z_{h}, r_{h}(x)\right)$.

Proof of Theorem 2.1: Since $\left(\frac{1}{a} f\right)^{\prime \prime}(a x)=a f^{\prime \prime}(x)$, after a suitable rescaling, we can suppose $f^{\prime \prime}(0)=1$.

Taking $\delta \in\left(0, \frac{1}{20}\right]$ from Lemma 2.3 we set $\sigma=\frac{\delta}{4}$. Suppose $z \in B(\sigma)$. There is $x_{0} \in\left[-\frac{\delta}{2}, \frac{\delta}{2}\right]$ such that

$$
\left|z-\left(x_{0}, f\left(x_{0}\right)\right)\right|=\operatorname{dist}(z,\{(x, f(x)): x \in \mathbb{R}\}) .
$$


Indeed, the graph of $f$ is a continuous curve, and, moreover

$$
\left|x_{0}\right| \leq|z|+\operatorname{dist}(z,\{(x, f(x)): x \in \mathbb{R}\}) \leq|z|+|z-(0,0)|=2|z| \leq \frac{\delta}{2} \text {. }
$$

Further

$$
\begin{aligned}
0 & =\left.\frac{\partial}{\partial x}\left(\left(x-z_{1}\right)^{2}+\left(f(x)-z_{2}\right)^{2}\right)\right|_{x=x_{0}} \\
& =2\left(x_{0}-z_{1}\right)+2\left(f\left(x_{0}\right)-z_{2}\right) f^{\prime}\left(x_{0}\right) \\
& =2\left(x_{0}-z_{1}, f\left(x_{0}\right)-z_{2}\right) \cdot\left(1, f^{\prime}\left(x_{0}\right)\right) .
\end{aligned}
$$

Thus, $z-\left(x_{0}, f\left(x_{0}\right)\right)$ is perpendicular to $\left(1, f^{\prime}\left(x_{0}\right)\right)$. Now, Proposition 2.2 and Lemma 2.3 conclude the proof.

\section{Local monotonicity of smooth curves}

In this section, we reformulate the main result about functions for $C^{2}$-curves, using the fact that they are locally graphs of $C^{2}$-functions.

Some notes on curves in $\mathbb{R}^{2}$. Let $I \subset \mathbb{R}$ be an open interval and $\gamma: I \rightarrow \mathbb{R}^{2}$ be a regular $C^{2}$-curve. We denote $\dot{\gamma}(t)=\left(\frac{\partial \gamma_{1}(t)}{\partial t}, \frac{\partial \gamma_{2}(t)}{\partial t}\right)$ and $\ddot{\gamma}(t)=\left(\frac{\partial^{2} \gamma_{1}(t)}{\partial t^{2}}, \frac{\partial^{2} \gamma_{2}(t)}{\partial t^{2}}\right)$ for $t \in I$. The curvature is defined by

$$
k_{\gamma}(t)=\frac{\left(M \dot{\gamma}(t)^{\top}\right)^{\top} \cdot \ddot{\gamma}(t)}{|\dot{\gamma}(t)|^{3}}, \quad \text { where } \quad M=\left(\begin{array}{rr}
0 & -1 \\
1 & 0
\end{array}\right) .
$$

Further, we can suppose $|\dot{\gamma}| \equiv 1$, which is obtained, for a regular curve, after a change of parameterization. In this case, $\left|k_{\gamma}(t)\right|=|\ddot{\gamma}(t)|$ and the geometrical meaning of the curvature is the following: for a fixed vector $v \in \mathbb{R}^{2}$, with $|v|=1$, and $t \in I$, let $\theta(t)$ be an angle such that $\cos \theta(t)=\dot{\gamma}(t) \cdot v, \sin \theta(t)=-\left(M \dot{\gamma}(t)^{\top}\right)^{\top} \cdot v$ and $\theta(t)$ is continuous with respect to $t$, then $\dot{\theta}(t)=k_{\gamma}(t)$.

Local monotonicity. Our aim is to prove

Theorem 3.1. Let $0<\varepsilon \leq K<\infty$ and $\omega:[0, \infty) \rightarrow[0, \infty)$ be a function satisfying $\lim _{t \rightarrow 0_{+}} \omega(t)=0$. Then there exists $\sigma>0$ such that $r \mapsto$ $\frac{\mu_{\gamma} B(z, r)}{r}$ is nondecreasing on the interval $(0, \min (\sigma,|z-\gamma(a)|,|z-\gamma(b)|))$ for every centre $z \in \mathbb{R}^{2}$ and every $C^{2}$-curve $\gamma:[a, b] \rightarrow \mathbb{R}^{2}$, where $-\infty<$ $a<b<\infty,|\dot{\gamma}| \equiv 1$, with the curvature $k_{\gamma}$ satisfying $k_{\gamma}(s) \in[\varepsilon, K]$ and $\left|k_{\gamma}(s)-k_{\gamma}(t)\right| \leq \omega(|s-t|), s, t \in(a, b)$.

In the following, standard calculations are checked to find the best radii. 
Lemma 3.2. Assume $\tilde{\delta}>0, \delta=\min \left(\frac{\tilde{\delta}}{2}, \frac{1}{20}\right), K \in(0, \infty)$. Let $I \subset\left(-\frac{\pi}{2 K}, \frac{\pi}{2 K}\right)$ be an open interval such that $0 \in I$ and $\gamma: I \rightarrow \mathbb{R}^{2}$ be $a C^{2}$-curve satisfying $\gamma(0)=(0,0), \dot{\gamma}(0)=(1,0),|\dot{\gamma}| \equiv 1, k_{\gamma}(I) \subset[0, K]$ and $\left|k_{\gamma}(t)-k_{\gamma}(0)\right| \leq \frac{k_{\gamma}(0)}{40}$ on $\left(-\frac{\tilde{\delta}}{K}, \frac{\tilde{\delta}}{K}\right) \cap I$.

Then $f(x)=\gamma_{2}\left(\gamma_{1}^{-1}(x)\right)$ is a $C^{2}$-function defined on $\gamma_{1}(I), f(0)=0$, $f^{\prime}(0)=0$ and $\left|f^{\prime \prime}(x)-f^{\prime \prime}(0)\right| \leq \frac{f^{\prime \prime}(0)}{20}$ on $\left(-\frac{\delta}{K}, \frac{\delta}{K}\right) \cap \gamma_{1}(I)$.

Proof: Let $\theta(t)$ be a continuously defined angle on $I$ such that $\cos \theta(t)=$ $\dot{\gamma}(t) \cdot \gamma(0)$ and $\sin \theta(t)=-\left(M \dot{\gamma}(t)^{\top}\right)^{\top} \cdot \gamma(0)$. Since

$$
|\theta(t)|=\left|\int_{0}^{t} \dot{\theta}(s) d s\right|=\left|\int_{0}^{t} k_{\gamma}(s) d s\right| \leq K|t|<\frac{\pi}{2},
$$

the function $f(x)=\gamma_{2}\left(\gamma_{1}^{-1}(x)\right)$ is a $C^{2}$-function on $\gamma_{1}(I)$ satisfying $f(0)=0, f^{\prime}(0)=0$ and

$$
f^{\prime \prime}(x)=k_{\gamma}\left(\gamma_{1}^{-1}(x)\right)\left(1+f^{\prime 2}(x)\right)^{\frac{3}{2}} .
$$

Finally, similarly as above, we obtain $\left|f^{\prime}(x)\right|=\left|\tan \theta\left(\gamma_{1}^{-1}(x)\right)\right|<\frac{1}{19}$ provided $|x| \leq \frac{\delta}{K}$. Thus

$$
\begin{aligned}
\left|f^{\prime \prime}(x)-f^{\prime \prime}(0)\right| & =\left|k_{\gamma}\left(\gamma_{1}^{-1}(x)\right)\left(1+f^{\prime 2}(x)\right)^{\frac{3}{2}}-k_{\gamma}(0)\right| \\
& \leq\left|k_{\gamma}\left(\gamma_{1}^{-1}(x)\right)-k(0)\right|+\left(\left(1+f^{\prime 2}(x)\right)^{\frac{3}{2}}-1\right)\left|k_{\gamma}\left(\gamma_{1}^{-1}(x)\right)\right| \\
& \leq \frac{1}{40} k_{\gamma}(0)+\left(\left(1+\frac{1}{19^{2}}\right)^{\frac{3}{2}}-1\right) \frac{41}{40} k_{\gamma}(0) \\
& \leq \frac{1}{20} k_{\gamma}(0)=\frac{1}{20} f^{\prime \prime}(0) .
\end{aligned}
$$

Proof of Theorem 3.1: Since $\lim _{t \rightarrow 0_{+}} \omega(t)=0$, there is $\tilde{\delta}>0$ such that $\omega(t) \leq \frac{\varepsilon}{40}$ on $(0, \tilde{\delta})$. We define $\delta=\min \left(\frac{\tilde{\delta}}{2}, \frac{1}{20}\right)$ and $\sigma=\frac{\delta}{K}$. Let $\gamma$ be a curve satisfying the assumptions of Theorem 3.1 and $z \in \mathbb{R}^{2}$. We set $\tau=\min (\sigma,|z-\gamma(a)|,|z-\gamma(b)|)$. If $\{\gamma(t): t \in[a, b]\} \cap B(z, \tau)=\emptyset$, then there is nothing to prove. Otherwise, there is a finite set $J \subset \mathbb{N}$ and closed intervals $I_{j}, j \in J$, such that

$$
\bigcup_{j \in J} I_{j}=\{t \in[a, b]: \gamma(t) \in B(z, \tau)\} .
$$

Now, for fixed $j \in J$, let us find $t_{j} \in I_{j}$ satisfying

$$
\left|\gamma\left(t_{j}\right)-z\right|=\operatorname{dist}\left(z,\left\{\gamma(t): t \in I_{j}\right\}\right) .
$$


Without loss of generality, suppose $\gamma\left(t_{j}\right)=(0,0)$ and $\dot{\gamma}\left(t_{j}\right)=(1,0)$. Then $z=z_{h}$, where $|h| \leq \tau$. Finally, after rescaling, observation (1), Lemma 3.2, and Proposition 2.2 give that $\left.\frac{1}{r} \mu_{\gamma}\right|_{\text {int } I_{j}} B(z, r)$ is nondecreasing on $(0, \tau)$. Therefore, $\frac{1}{r} \mu_{\gamma} B(z, r)=\left.\sum_{j \in J} \frac{1}{r} \mu_{\gamma}\right|_{\operatorname{int}_{I_{j}}} B(z, r)$ is also nondecreasing on $(0, \tau)$.

\section{Example}

Theorem 4.1. There is a strictly convex function $f \in C^{\infty}(\mathbb{R})$ and a sequence of nonempty intervals $I_{k} \subset \mathbb{R}, k \in \mathbb{N}$, $\operatorname{dist}\left(I_{k}, 0\right) \rightarrow 0$, such that $r \mapsto \frac{\mu_{f} B((0, f(0)), r)}{r}$ is decreasing on every $I_{k}$.

Before constructing such a function, we prove the following two lemmas

Lemma 4.2. There are $a_{0}>0$ and $\varepsilon_{0}>0$ such that $r \mapsto \frac{\mu_{f} B(r)}{r}$ is decreasing on $\left(r_{0}\left(2^{4} \varrho\right), r_{0}\left(2^{5} \varrho\right)\right)$ for every $\varrho>0, a \in\left(0, a_{0}\right], \varepsilon \in$ $\left[0, \varepsilon_{0}\right]$ and every even continuous function $f$ satisfying $f(0)=0, f(2 \varrho) \in$ $[0,(1+2 \varepsilon) a \varrho]$ and $f^{\prime}(x) \in[a,(1+\varepsilon) a]$ on $\left[2 \varrho, 2^{5} \varrho\right]$.

Proof: Rescaling we suppose $\varrho=1$. For $a>0, \xi \in\left[0, \frac{3}{2}\right]$ and $t \in\left[2^{4}, 2^{5}\right]$ we define

$$
\begin{aligned}
\Psi_{a, \xi}(t)= & \sqrt{1+a^{2}}\left(t^{2}+a^{2}(\xi+t-2)^{2}\right) \\
& -\left(t+a^{2}(\xi+t-2)\right)\left(\sqrt{4+a^{2} \xi^{2}}+(t-2) \sqrt{1+a^{2}}\right) \\
= & a^{2} \xi^{2} \sqrt{1+a^{2}}-2 a^{2} \xi \sqrt{1+a^{2}}-2 a^{2} \xi \sqrt{1+\frac{a^{2} \xi^{2}}{4}}+4 a^{2} \sqrt{1+\frac{a^{2} \xi^{2}}{4}} \\
& -\left(-a^{2} \xi \sqrt{1+a^{2}}-2 \sqrt{1+a^{2}}+2 \sqrt{1+\frac{a^{2} \xi^{2}}{4}}+2 a^{2} \sqrt{1+\frac{a^{2} \xi^{2}}{4}}\right) t \\
= & \left(1-\frac{t}{4}\right)\left(a^{2} \xi^{2}-4 a^{2} \xi+4 a^{2}+o\left(a^{2}\right)\right) \\
\leq & \left(1-\frac{t}{4}\right) \frac{1}{4} a^{2}+o\left(a^{2}\right) .
\end{aligned}
$$


Now, if $a>0$ and $\varepsilon \in\left(0, \frac{1}{4}\right)$ are sufficiently small, then we have

$\Psi_{a, \varepsilon, \xi}(t)=\sqrt{1+(1+\varepsilon)^{2} a^{2}}\left(t^{2}+a^{2}(\xi+(1+\varepsilon)(t-2))^{2}\right)$

$$
\begin{array}{r}
-\left(t+a^{2}(\xi+t-2)\right)\left(\sqrt{4+a^{2} \xi^{2}}+(t-2) \sqrt{1+a^{2}}\right) \\
\leq \Psi_{a, \xi}(t)+\left(\sqrt{1+(1+\varepsilon)^{2} a^{2}}-\sqrt{1+a^{2}}\right) 2 t^{2} \\
+2 a^{2} \varepsilon(t-2)(2 \xi+(2+\varepsilon)(t-2))
\end{array}
$$

$<0$.

Finally, if we set $\xi=\frac{f(2 \varrho)}{a \varrho}$, then the parameterization used in Lemma 2.4 gives

$$
\begin{aligned}
\left.\frac{\partial}{\partial r} \frac{\mu_{f} B(r)}{r}\right|_{r=r_{0}(t)} & =\left.\frac{1}{r^{2}}\left(\frac{\partial \mu_{f} B(r)}{\partial r} r-\mu_{f} B(r)\right)\right|_{r=r_{0}(t)} \\
& =\frac{1}{r_{0}^{2}(t)}\left(2 \frac{\sqrt{1+f^{\prime 2}(t)} r_{0}(t)}{t+f(t) f^{\prime}(t)}-\mu_{f} B\left(r_{0}(t)\right)\right) \\
& \leq \frac{2}{r_{0}^{2}(t)} \frac{\Psi_{a, \varepsilon, \xi}(t)}{t+f(t) f^{\prime}(t)} \\
& <0 .
\end{aligned}
$$

Lemma 4.3. Let $k \in \mathbb{N}, \varrho>0, \theta>1$ and $\delta>0$. Then there exists an even $C^{\infty}$-function $\varphi$ such that $\varphi(x)=0$ on $[0, \varrho], f$ is strictly convex on $(\varrho, \theta \varrho)$, affine on $[\theta \varrho, \infty)$ and $\left|\mathrm{D}^{\mathrm{i}} f(x)\right| \leq \delta$ for every $1 \leq i \leq k$ and every $x \in \mathbb{R}$.

Proof: We define

$$
\psi(x)= \begin{cases}0, & x \in(-\infty,-1], \\ \exp \left(-\frac{1}{(1-x)^{2}}\right), & x \in[-1,0], \\ e^{-1}, & x \in[0, \infty) .\end{cases}
$$

Now, we set $\Psi(x)=\int_{-\infty}^{x} \psi(t) d t$. Hence, if $\alpha, \beta, \sigma \in \mathbb{R}$ are suitably chosen, then $\varphi(x)=\alpha \Psi(\beta|x|+\sigma)$ is the required function.

Proof of Theorem 4.1: We find two sequences of even $C^{\infty}$-functions. The function $f_{0}$ satisfies $f_{0}(x)=0$ on $[0,1]$ and $f_{0}$ is strictly convex on $(1, \infty)$. We set $g_{0} \equiv 0$. 
Having $a>0$ and $0<\varepsilon \leq 1$ from Lemma 4.2, we use Lemma 4.3 to obtain the functions $f_{1}$ and $g_{1}$ satisfying $f_{1}(x)=0$ on $\left[0,2^{-5}\right], f_{1}$ is strictly convex on $\left(2^{-5}, 2^{-4}\right), f_{1}^{\prime}(x)=a_{1}$ on $\left[2^{-4}, \infty\right)$, where $0<a_{1} \leq$ $\min \left(a, 2^{-6}\right), g_{1}(x)=0$ on $\left[0,2^{-4}\right], g_{1}$ is strictly convex on $\left(2^{-4}, 1\right), g_{1}$ is affine on $[1, \infty)$ and $g_{1}^{\prime}(x) \leq \frac{\varepsilon a_{1}}{4}$ for all $x \geq 0$.

Having found $f_{k-1}$ and $g_{k-1}$, let $f_{k}$ and $g_{k}$ satisfy $f_{k}(x)=0$ on $\left[0,2^{-5 k}\right]$, $f_{k}$ is strictly convex on $\left(2^{-5 k}, 2^{-5 k+1}\right), f_{k}^{\prime}(x)=a_{k}$ on $\left[2^{-5 k+1}, \infty\right)$, where $a_{k}>0,\left|\mathrm{D}^{\mathrm{i}} f_{k}(x)\right| \leq \min \left(\frac{\varepsilon}{4} a_{k-1}, 2^{-6 k}\right)$ for all $x \geq 0$ and every $1 \leq i \leq k$, $g_{k}(x)=0$ on $\left[0,2^{-5 k+1}\right], g_{k}$ is strictly convex on $\left(2^{-5 k+1}, 2^{-5(k-1)}\right)$, $g_{k}$ is affine on $\left[2^{-5(k-1)}, \infty\right)$ and $\left|\mathrm{D}^{\mathrm{i}} g_{k}(x)\right| \leq \frac{\varepsilon}{4} a_{k}$ for all $x \geq 0$ and every $1 \leq i \leq k$.

Now, we show that $f=\sum_{k=0}^{\infty} f_{k}+g_{k}$ is the required function. Let us prove that $f \in C^{\infty}(\mathbb{R})$. For any $x>0$, there is at most one function in the sum to have generally non-zero derivatives of a higher order than one. Moreover, the estimates of $\left|\mathrm{D}^{\mathrm{i}} f_{k}(x)\right|$ and $\left|\mathrm{D}^{\mathrm{i}} g_{k}(x)\right|$ imply $\mathrm{D}^{\mathrm{i}+1} f(0)=0$ for all $i \in \mathbb{N}$. Further, the sum of the first derivatives is always finite.

Finally, if $x \in\left[2^{-5 k+1}, 2^{-5(k-1)}\right], k \in \mathbb{N}$, then we have

$a_{k} \leq f^{\prime}(x)=\sum_{n=k}^{\infty} f_{n}^{\prime}(x)+g_{n}^{\prime}(x) \leq a_{k} \sum_{n=k}^{\infty}\left(\frac{\varepsilon}{4}\right)^{n-k}+\left(\frac{\varepsilon}{4}\right)^{n-k+1} \leq(1+\varepsilon) a_{k}$.

Hence, the non monotonicity on the $r_{0}(\cdot)$-image of each interval $\left(2^{-5 k+4}, 2^{-5 k+5}\right), k \in \mathbb{N}$, follows from Lemma 4.2 .

Acknowledgement. The research is supported by grants GA ČR 201/ 03/0931 and MSM 113200007. The author is grateful to Jan Kolár for fruitful discussions. The author would like to thank the referee for the recommendations that considerably simplified the proof of Proposition 2.2 and improved the smoothness in Theorem 4.1.

\section{References}

[1] W. K. Allard, On the first variation of a varifold, Ann. of Math. (2) 95 (1972), 417-491.

[2] R. ČERnÝ AND J. KolÁř, Monotone measures with bad tangential behaviour in the plane, in preparation.

[3] R. Černý and J. Kolár̆, Monotone measures with bad tangential behaviour in $R^{3}$, in preparation.

[4] D. Preiss, Geometry of measures in $\mathbb{R}^{n}$ : distribution, rectifiability, and densities, Ann. of Math. (2) 125(3) (1987), 537-643. 
[5] L. Simon, "Lectures on geometric measure theory", Proceedings of the Centre for Mathematical Analysis, Australian National University 3, Australian National University, Centre for Mathematical Analysis, Canberra, 1983.

Department of Mathematical Analysis

Faculty of Mathematics and Physics

Charles University

Sokolovská 83

18675 Praha 8

Czech Republic

E-mail address: rcerny@karlin.mff.cuni.cz

Primera versió rebuda el 28 d'abril de 2003,

darrera versió rebuda el 19 de novembre de 2003. 